\title{
Anterolateral approach for thyroid swellings: impact on postoperative voice outcomes
}

\author{
Bin Zhang, Bin Lv, Nan Liu \\ Department of Thyroid Surgery, General Surgery, Qilu Hospital of Shandong University, Jinan, China \\ Contributions: (I) Conception and design: All authors; (II) Administrative support: N Liu; (III) Provision of study materials or patients: B Lv, N Liu; \\ (IV) Collection and assembly of data: B Zhang; (V) Data analysis and interpretation: B Zhang; (VI) Manuscript writing: All authors; (VII) Final \\ approval of manuscript: All authors. \\ Correspondence to: Nan Liu. Department of Thyroid Surgery, General Surgery, Qilu Hospital of Shandong University, Jinan 250012, China. \\ Email: liunan0308@hotmail.com.
}

Background: The sternothyroid muscle (ST) is routinely divided during grossly enlarged thyroid surgery to facilitate gland exposure. However, the ST is considered to play a role in controlling of pitch in the human voice. In this prospective cohort study, we described an anterolateral approach (AA) by which it was easy to expose the enlarged gland while preserving the ST, and evaluated the impact of this approach on vocal outcomes after total thyroidectomy.

Methods: The AA was performed on eligible patients from October 2018 to January 2020 in our department. Consecutive cases hospitalized from March 2020 and followed up to June 2021 who received total thyroidectomy via the midline approach (MA) served as controls. All participants underwent voice analysis before the operation and at 2 weeks and 3 months after the surgery. In 26 patients from AA group, strap muscles were mobilized along the anterior margin of both sternocleidomastoid muscles (SCMs) until $3 \mathrm{~cm}$ above the sternal notch followed by thyroidectomy, and their voice outcomes were compared to those of 36 patients from the MA group with a bilateral division of ST.

Results: Distribution of age, gender, specimen size, thyroid histopathology and laryngeal nerve identification rates did not differ significantly between groups. At 2 weeks post-surgery, objective assessment of voice change rate indicated no significant difference between groups, whereas the difference appeared to be partially manifest 3 months later. In the single-arm study, the AA group showed a decrease of maximum and fundamental pitch frequency 2 weeks after surgery but showed no difference in fundamental frequency after 3 months. The MA group continued to show a mild decrease in maximum and fundamental frequency at 3 months after surgery.

Conclusions: The AA affords sufficient visualization of the superior thyroid vessels and external branch of the superior laryngeal nerve (EBSLN) without sacrificing the integrity of the strap muscles. This approach is a feasible solution for selective difficult thyroid surgery and could reduce the postoperative changes in pitch of voice.

Keywords: Surgical technique; anterolateral approach (AA); voice; thyroidectomy

Submitted Jul 31, 2021. Accepted for publication Oct 15, 2021.

doi: $10.21037 / g s-21-510$

View this article at: https://dx.doi.org/10.21037/gs-21-510

\section{Introduction}

Significant thyroid swelling is one of the main indications for surgery and a risk factor for laryngeal nerve injury $(1,2)$. Different modes of compression symptoms can present, such as globus sensation, hoarse voice, dysphagia, and dyspnea, which are often exacerbated when supine. From a surgical perspective, a grossly enlarged gland often distorts the relative relationship between anatomic structures, 
including the external branch of the superior laryngeal nerve (EBSLN) and the upper thyroid pole. The practical intervention for managing an enlarged thyroid involves early complete mobilization of the superior pole and the division of isthmus from the trachea to achieve great mobility of the thyroid lobe (3). However, it is difficult to perform lateral and downward retraction of an enlarged upper pole, which is restricted by the strap muscles and large superior thyroid vessels, to enter the avascular space between the gland and cricothyroid. Thus, cutting of the sternothyroid muscle (ST) is routinely performed, in combination with sternohyoid muscle (SH) division when necessary (4). Voice preservation is a key concern of both surgeon and patients undergoing thyroid surgery. Voice disturbances after thyroidectomy where the laryngeal nerves have been functionally safeguarded may be caused by damage to any of the intrinsic cervical structures involved in the complex phonation system (5). The strap muscles may be damaged by denervation, forced lateral retraction or complete transection.

During an open thyroidectomy, different approaches can be used depending on the patient's type of thyroid growth and choice (6). In this study, we have reported an alternative approach, the anterolateral approach (AA), which is performed between the sternocleidomastoid muscles (SCMs) and strap muscles, and evaluated the impact of functional preservation of the strap musculature on voice outcomes. We present the following article in accordance with the STROBE reporting checklist (available at https:// dx.doi.org/10.21037/gs-21-510).

\section{Methods}

\section{Participants}

This was a prospective data analysis of thyroid swelling patients who underwent primary total thyroidectomy at the Qilu Hospital of Shandong University between October 2018 and June 2021. The AA was performed on consecutive patients from October 2018 to January 2020, and eligible cases who were hospitalized from March 2020, received thyroidectomy via midline approach (MA), and followed up until June 2021 served as the control group. Before surgery, all participants underwent high-resolution ultrasound, computerized tomography (CT), laryngoscopy, and acoustic examination. During postoperative follow-up, the participants' voice outcomes and laryngoscopic views were assessed at 2 weeks and 3 months after the operation. The study was conducted in accordance with the Declaration of Helsinki (as revised in 2013). The study was approved by Committee on Scientific Research of Shandong University Qilu Hospital (No. 2018149) and informed consent was taken from all individual participants. The design of the study is shown in the flow chart (Figure 1).

\section{Inclusions and exclusions}

All eligible patients had presented with thyroid swelling with a relatively symmetrical type of thyroid growth where the maximum dimension of the horizontal or vertical axes exceeded the medial border of the SCM and upper border of thyroid cartilage as determined by CT images, and in which a maximum of $3 \mathrm{~cm}$ of the inferior pole was in a retrosternal position. Patients were excluded if they had any of the following: previous neck surgery; history of alcohol drinking or smoking; a pathology in the laryngoscopy before operation; iatrogenic damage to the laryngeal nerves or cricothyroid muscles.

\section{Surgical technique in thyroidectomy}

Thyroidectomy was conducted according to the standardized or modified technique as follows. A low "collar" transcervical incision was made with adequate skin flap elevation. The incision could be extended posterolaterally to the SCM to enable wider access to the lateral margin of large tumors. The ST was divided bilaterally during surgery via $\mathrm{MA}$, and the $\mathrm{SH}$ was retracted away from the midline. The upper part of the transected ST was removed to expose the superior pole further, and end-to-end suturing of the divided muscle was not conducted. During surgery via AA, the strap muscles were mobilized along the anterior margin of SCM to $3 \mathrm{~cm}$ above the sternal notch to preserve innervation of the strap muscles (7). We performed a meticulous dissection in every ansa cervicalis and confirmed its integrity via corresponding strap muscle contraction using electro-stimulation before and after the thyroid dissection. Then by combining the lateral and medial retraction of the strap muscles, we provided an adequate visual field of the lateral or medial aspect of the thyroid lobe (Figure 2). Exposure of the superior pole was achieved by pulling the gland in an inferior and medial direction to the space developed between the SCM and straps, followed by individual ligation of the superior pole vessels. With intraoperative nerve monitoring (IONM), the EBSLN was visualized or monitored by stimulating the nerve from the 


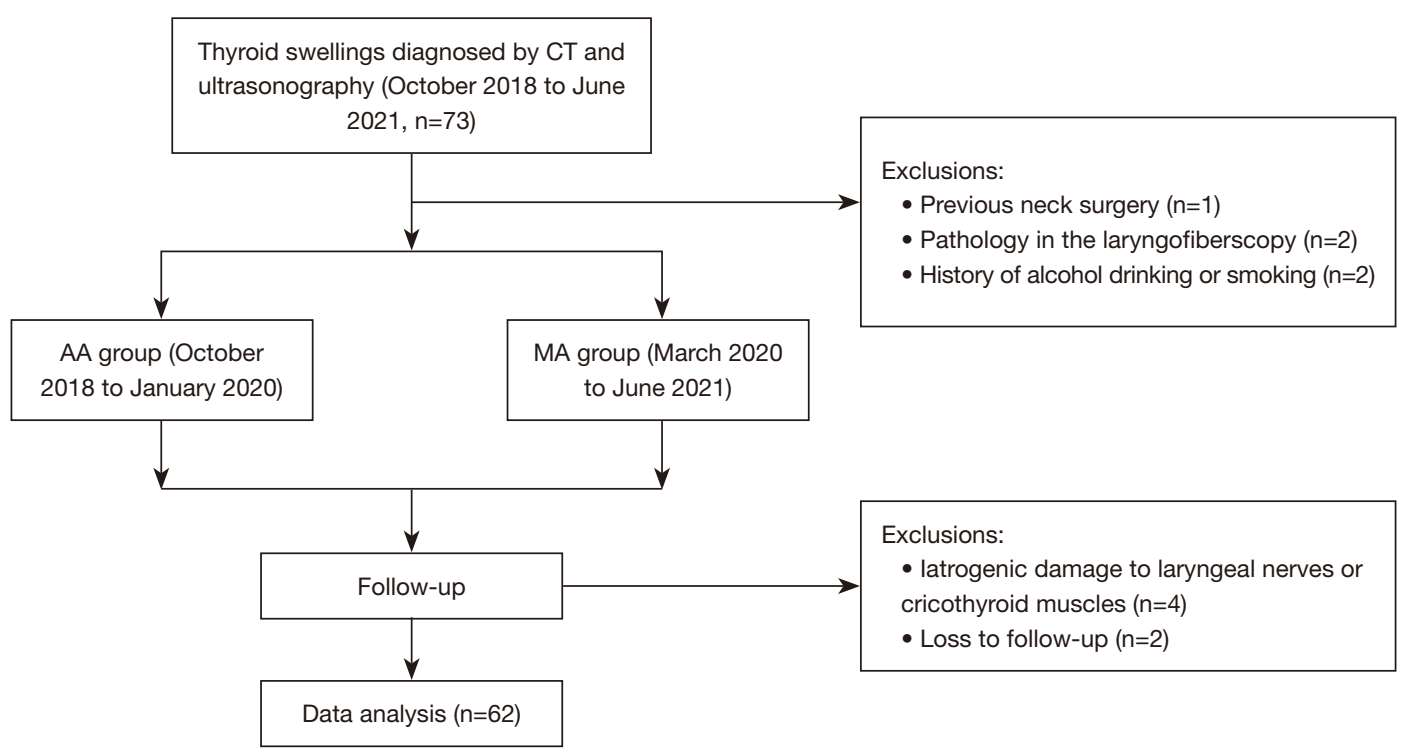

Figure 1 The study design flow chart. CT, computerized tomography; AA, anterolateral approach; MA, midline approach.
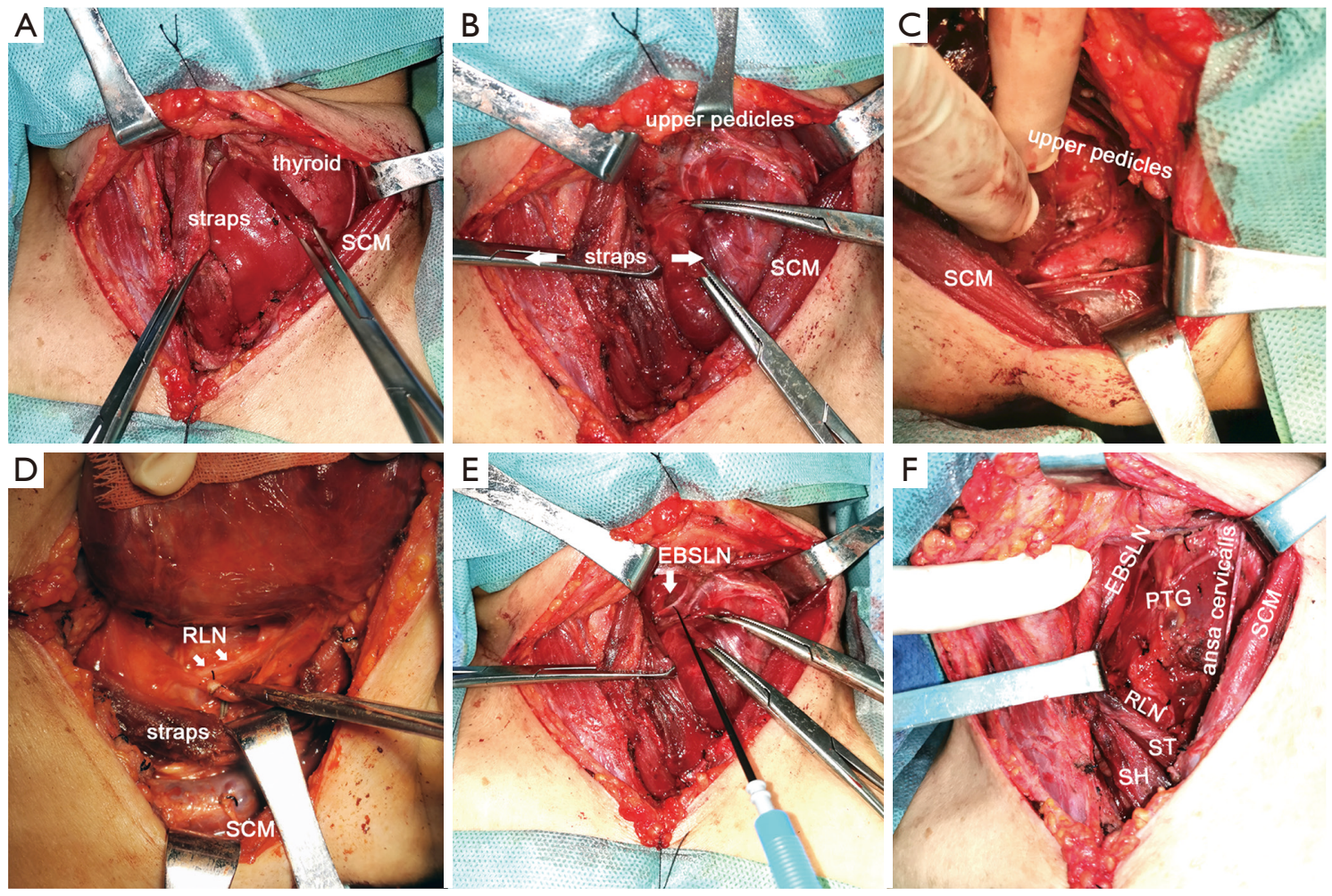

Figure 2 Thyroid surgeries via AA. (A) Strap muscles were freed from the anterior margin of SCMs and the thyroid gland; (B) the strap muscles were retracted laterally or medially as required to match the exposure of surgical field for total thyroidectomy; (C) lateral access to the upper pedicles; (D) RLN dissection; (E) external branch of superior laryngeal nerve was maintained and confirmed by electrostimulation; (F) good preservation of the ST and its innervation. SCM, sternocleidomastoid muscle; SH, sternohyoid muscle; ST, sternothyroid muscle; PTG, parathyroid gland; RLN, recurrent laryngeal nerve; EBSLN, external branch of superior laryngeal nerve; AA, anterolateral approach. 
point proximal to the excision of the superior pole vessels with an integral twitch of the cricothyroid muscles, or with an electromyographic (EMG) glottic response recorded through electrodes on an endotracheal tube after safe ligation of the superior pole vessels. The recurrent laryngeal nerve (RLN) was dissected in a retrograde or anterograde fashion by lateral or medial approach after the upper pole was freed. A $>50 \%$ decrease in amplitude paralleled by a $>10 \%$ increase in latency of EMG signals or loss of signal recorded during IONM which failed to restore during surgery were deemed iatrogenic RLN damage.

\section{Acoustic analysis}

Acoustic analysis was performed on all eligible patients by a speech pathologist who was blinded to the research hypothesis using DiVAS program (XION medical, Berlin, Germany). The software presented the notes of vocal capacity including minimum pitch frequency $\left(\mathrm{F}_{0}-\mathrm{low}\right)$, maximum pitch frequency $\left(\mathrm{F}_{0}\right.$-high), the range of frequency ( $\mathrm{F}_{0}$-range), lowest intensity (I-low), highest intensity (I-high), and the range of intensity (I-range). Another measure of vocal capacity is the maximum phonation time (MPT) which was obtained during phonation of /a:/ at a constant pitch and intensity after full inspiration. The maximum value among 3 consecutive examinations of MPT was taken as the measurement. The parameters of voice stability, including fundamental frequency $\left(\mathrm{F}_{0}\right)$, jitter and shimmer were also evaluated. The 6 most applicable and comparable parameters among those recordings were taken for analysis: $\mathrm{F}_{0}, \mathrm{~F}_{0}$-low, $\mathrm{F}_{0}$-high, MPT, jitter and shimmer. It is generally accepted that adult men have a low-pitched voice and women a high-pitched voice (8). We, therefore, analyzed the measurements of $\mathrm{F}_{0}, \mathrm{~F}_{0}$-low and $\mathrm{F}_{0}$-high separately by gender to reduce gender-related bias. The voice change from baseline to postoperatively was calculated by the formula:

$$
\Delta=100 \% \times(B-A) / A
$$

where $A$ and $B$ are preoperative and postoperative values at each follow-up time point, with each participant serving as his or her own control.

\section{Perceptual analysis}

Perceptual analysis was conducted by 2 trained assessors of our center, including the speech pathologist and a laryngologist who was blinded to the research hypothesis using the grade, roughness, breathiness, asthenia, strain (GRBAS) scale proposed by Hirano, with the grade (G) appearing to be the most reliable parameter (9). In the present study, only the $G$ parameter was included and judged on a scale of 0 to 3 ( 0 , absent; 1 , mild; 2 , moderate; 3 , severe). The inter-rater reliability evaluated by Spearman's rank correlation coefficient showed significant correlation $(\mathrm{r}=0.83, \mathrm{P}<0.01)$, and the mean rating score between 2 raters was calculated and compared between groups.

\section{Statistical analysis}

Statistical analyses were conducted with SPSS software (IBM SPSS Statistics, RRID: SCR_019096) version 19.0. The estimated volume of each thyroid lobe was calculated based on ellipsoid formula:

$$
\pi / 6 \times(\text { length } \times \text { width } \times \text { thickness })
$$

An independent sample $t$-test was performed to compare the numeric data between 2 groups, and paired samples $t$-test was conducted to analyze the postoperative change of the parameters in each group. Fisher's exact test was used for the analysis of categorical data. A 2 -sided $\mathrm{P}$ value $<0.05$ was considered statistically significant.

\section{Results}

Of the initial 73 patients who were enrolled from October 2018 and completed follow-up before June 2021, 5 were excluded due to various preoperative conditions that affected voice quality. A further 2 participants were lost to follow-up due to unavailability to connect, and 4 more were excluded because of laryngeal nerve or cricothyroid muscle damage. The remaining participants had no fixation and impaired mobility of the vocal folds during postoperative laryngoscopic examinations. Distribution of age, gender, thyroid histopathology, specimen size, and laryngeal nerve identification rates did not differ significantly between groups (Table 1). Out of 26 patients in AA group, histopathology was multinodular goiter (MNG) in 15 $(57.7 \%)$, lymphocytic thyroiditis in $2(7.7 \%)$, toxic goiter in $4(15.4 \%)$, MNG with follicular carcinoma (FTC) in 1 (3.8\%) and MNG with papillary microcarcinoma (MPTC) in $4(15.4 \%)$ patients. Among 36 patients of MA group, 19 (52.8\%) patients had MNG, $1(2.8 \%)$ had lymphatic thyroiditis, 5 (13.9\%) had toxic goiter, 3 (8.3\%) had MNG with FTC and 8 (22.2\%) had MNG with MPTC. Though there was no operative mortality and major complications 
Table 1 Demographic and clinical characteristics of study groups

\begin{tabular}{|c|c|c|c|}
\hline Variables & AA group $(n=26)$ & MA group $(n=36)$ & $\mathrm{P}$ \\
\hline Sex (female/male) & $23 / 3$ & $31 / 5$ & $1.00^{\dagger}$ \\
\hline Histology (benign/unexpected malignancy) & $21 / 5$ & $25 / 11$ & 0.32 \\
\hline Maximum thyroid specimen size $(\mathrm{cm})$ & $8.41 \pm 0.55$ & $8.69 \pm 0.65$ & 0.08 \\
\hline EBSLN identified during operation (\%) & 92.3 & 88.9 & 0.53 \\
\hline RLN identified during operation (\%) & 100 & 100 & - \\
\hline Operating time (min) & $149.96 \pm 9.58$ & $132.30 \pm 11.15$ & $<0.01$ \\
\hline \multicolumn{4}{|l|}{ Preoperative baseline of acoustic parameters } \\
\hline $\mathrm{F}_{0}$-high, female $(\mathrm{Hz})$ & $462.83 \pm 67.12$ & $448.10 \pm 39.96$ & 0.32 \\
\hline $\mathrm{F}_{0}$-high, male $(\mathrm{Hz})$ & $406.67 \pm 102.83$ & $382.60 \pm 58.57$ & 0.68 \\
\hline $\mathrm{F}_{0}$-low, female $(\mathrm{Hz})$ & $180.04 \pm 20.61$ & $168.61 \pm 27.02$ & 0.10 \\
\hline $\mathrm{F}_{0}$-low, male $(\mathrm{Hz})$ & $113.00 \pm 17.44$ & $101.00 \pm 7.65$ & 0.21 \\
\hline MPT (s) & $16.40 \pm 4.83$ & $14.91 \pm 3.57$ & 0.19 \\
\hline Jitter (\%) & $0.74 \pm 0.17$ & $0.79 \pm 0.15$ & 0.22 \\
\hline Shimmer (\%) & $2.29 \pm 0.42$ & $2.32 \pm 0.36$ & 0.80 \\
\hline G score & $0.37 \pm 0.57$ & $0.44 \pm 0.56$ & 0.67 \\
\hline
\end{tabular}

Independent sample $t$-test was used to compare the numeric data between two groups. ${ }^{\dagger}$, Fisher's exact test. AA, anterolateral approach; $M A$, midline approach; $F_{0}$, fundamental frequency; $F_{0}$-high, maximum pitch frequency; $F_{0}$-low, minimum pitch frequency; MPT, maximum phonation time; $\mathrm{G}$, the parameter grade of GRBAS scale.

such as hematoma or permanent hypoparathyroidism after all surgeries, vocal function measurements showed significant changes in various acoustic parameters in both groups (Table 2). In the early postoperative period, the most important changes were lowering the habitual pitch, decreased vocal performance with reduced $\mathrm{F}_{0^{-}}$ high, and increased perturbation parameters such as jitter and shimmer (all $\mathrm{P}<0.05$ ). At 3 months post-surgery, the participants with divided ST were unable to reach the same habitual and high pitch frequency as preoperatively $(\mathrm{P}<0.05)$. Voice improvement was detected in the habitual pitch and perturbation parameters after 3 months in the AA group, but the reduction of $\mathrm{F}_{0}$-high remained statistically evident $(\mathrm{P}<0.05)$. Between-group comparisons of acoustic changes from the baseline indicated the earlier or better recovery of $\mathrm{F}_{0}$ and $\mathrm{F}_{0}$-high in the AA group than in the MA group $\left(\mathrm{P}<0.05\right.$; Table 3). However, the minor disparity of $\mathrm{F}_{0}$ and $\mathrm{F}_{0^{-}}$ high change percent between groups could not be mirrored by subjective parameter $\mathrm{G}(\mathrm{P}>0.05)$, which only described the grade of hoarseness but rather the vocal capability. Additionally, the mean operating time of the AA group was longer than that of the MA group $(\mathrm{P}<0.05)$.

\section{Discussion}

Thyroid swelling is a problem of extensive magnitude all over the world. Those affected often experience generalized enlargement of the thyroid gland and the final pathological diagnosis include Graves' disease, Hashimoto's thyroiditis, diffuse or MNG, thyroid adenoma or FTC. Thyroid swellings are 4 times more common in females than in males. Nodular goiter is the most common cause (10). Significant thyroid enlargement exhibits airway or esophagus pressure symptoms and cosmetic deformity, which is the main indication for 
Table 2 Comparison of acoustic measurements according to pre- and postoperative periods in each cohort

\begin{tabular}{|c|c|c|c|c|c|c|c|c|}
\hline Variables & \multicolumn{4}{|c|}{ AA group } & \multicolumn{4}{|c|}{ MA group } \\
\hline $\mathrm{F}_{0}$, female $(\mathrm{Hz})$ & $245.22 \pm 30.71$ & $<0.01$ & $262.70 \pm 29.74$ & 0.06 & $227.16 \pm 35.85$ & $<0.01$ & $248.58 \pm 31.97$ & $<0.01$ \\
\hline $\mathrm{F}_{0}$-high, female $(\mathrm{Hz})$ & $379.26 \pm 54.76$ & $<0.01$ & $443.52 \pm 66.04$ & $<0.01$ & $350.03 \pm 49.55$ & $<0.01$ & $415.61 \pm 40.55$ & $<0.01$ \\
\hline $\mathrm{F}_{0}$-low, female $(\mathrm{Hz})$ & $169.91 \pm 20.30$ & $<0.01$ & $177.43 \pm 21.53$ & 0.18 & $150.29 \pm 31.32$ & $<0.01$ & $164.87 \pm 21.00$ & 0.06 \\
\hline $\mathrm{F}_{0}$-high, male $(\mathrm{Hz})$ & $334.33 \pm 96.72$ & 0.03 & $381.67 \pm 87.81$ & 0.11 & $322.60 \pm 55.31$ & $<0.01$ & $351.40 \pm 49.89$ & $<0.01$ \\
\hline $\mathrm{F}_{0}$-low, male $(\mathrm{Hz})$ & $106.00 \pm 21.00$ & 0.29 & $111.00 \pm 13.75$ & 0.70 & $94.80 \pm 6.30$ & 0.24 & $98.80 \pm 7.12$ & 0.62 \\
\hline MPT (s) & $15.63 \pm 4.90$ & 0.07 & $16.82 \pm 4.26$ & 0.42 & $14.03 \pm 3.18$ & 0.06 & $14.69 \pm 3.39$ & 0.51 \\
\hline Jitter (\%) & $0.87 \pm 0.16$ & $<0.01$ & $0.76 \pm 0.14$ & 0.39 & $0.95 \pm 0.20$ & $<0.01$ & $0.82 \pm 0.12$ & 0.25 \\
\hline
\end{tabular}

Paired samples $t$-test was performed to analyze the postoperative change of the parameters in each group. P1 and P3, comparison of preoperative and early postoperative periods; $\mathrm{P} 2$ and $\mathrm{P} 4$, comparison of preoperative and late postoperative periods. Post-op, postoperative. AA, anterolateral approach; $M A$, midline approach; $F_{0}$, fundamental frequency; $F_{0}$-high, maximum pitch frequency; $F_{0}$-low, minimum pitch frequency; MPT, maximum phonation time; $\mathrm{G}$, the parameter grade of GRBAS scale.

Table 3 Acoustic changes from baseline in patients with and without ST division

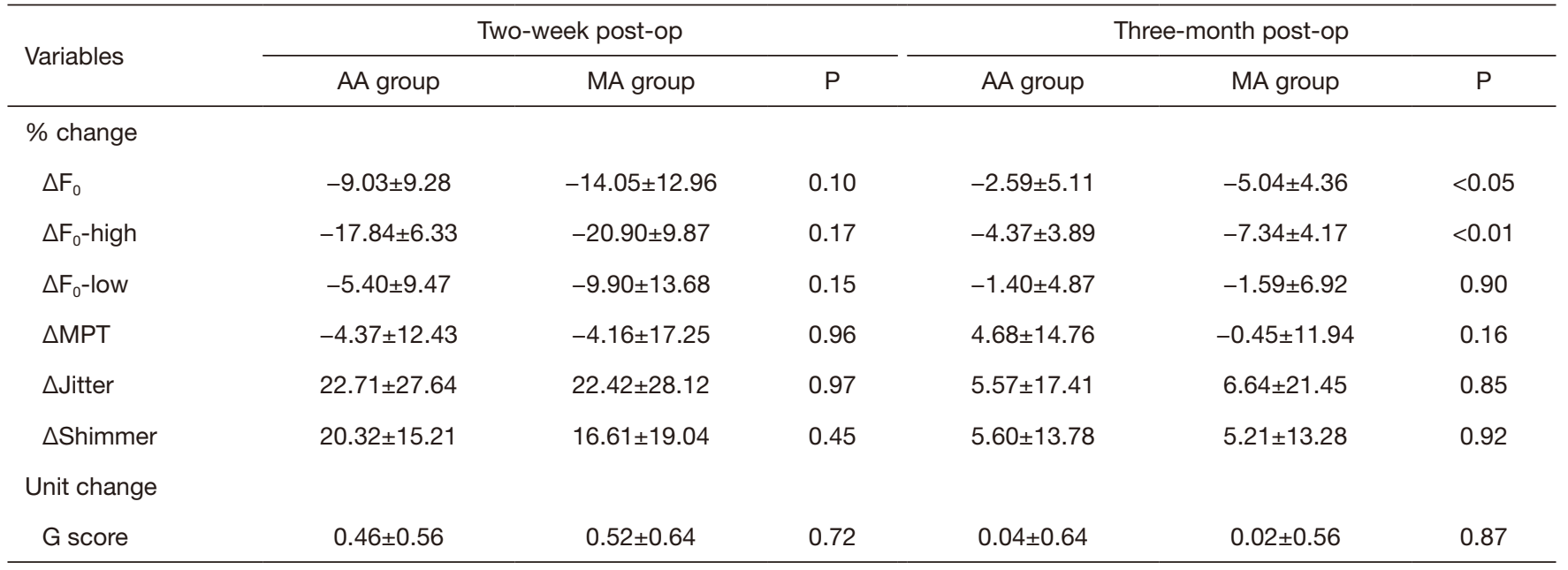

Independent sample $t$-test was used to compare the numeric data between two groups. The calculation formula of $\Delta$ was noted in the method. ST, sternothyroid muscle; Post-op, postoperative; AA, anterolateral approach; MA, midline approach; $F_{0}$, fundamental frequency; $F_{0}$-high, maximum pitch frequency; $F_{0}$-low, minimum pitch frequency; MPT, maximum phonation time; $G$, the parameter grade of GRBAS scale.

surgery and a simultaneous risk factor for laryngeal nerve injury. During these difficult thyroid surgeries, the RLN can be identified using different approaches depending on the morphology of thyroid growth: there are lateral, inferior, superior, and medial approaches. For convenience, most lesions are removed through a MA, and most have a lateral retraction of the SH after the division of the ST without restoration of its continuity. However, the strap muscles may participate in gross movement of the larynx during swallowing and adjust the larynx's positioning during vocalization, among which the ST, attaching cranially to the anterolateral aspect of the thyroid cartilage, causes laryngeal depression (11). 
Sonninen reported that elevation or depression of the larynx is considered to shorten or lengthen the vocal folds and regulates pitch by changing the relation of the cricoid to the thyroid cartilage (12). Furthermore, many researchers have proposed the $\mathrm{F}_{0}$ raising role of the $\mathrm{ST}$ in manipulating thyroid cartilage to rotate downward around the cricothyroid joint (13). Some reports have argued that the $\mathrm{SH}$ and $\mathrm{ST}$ contraction causes the laryngotracheal downward pull, which ultimately translates into adjusting the voice frequency (14).

Voice performance after thyroid surgery is one of the main concern relating to patients' emotional state and quality of life. Phonation is produced by the complex anatomy and physiology of the larynx, trachea, strap muscles and nerve innervation. The importance of factors other than laryngeal nerve injury contributing to voice changes after thyroidectomy is underestimated. With comprehensive voice analysis, a contributory role of the $\mathrm{ST}$ in voice production has been suggested by this study. Acoustic changes from the preoperative baseline indicated an earlier recovery of $\mathrm{F}_{0}$ and $\mathrm{F}_{0}$-high in the AA group than in the MA group in a 3-month follow-up period. However, it was unclear whether the disparity among groups remains statistically significant in a long-term period. Sinagra demonstrated that $F_{0}$ gradually recovered in the fourth and sixth postoperative months but scarcely reached preoperative levels (15). Hong showed that the habitual pitch and speaking frequency range were significantly decreased 1 and 3 months after thyroid surgery but not at 6 months (16). We believe early voice improvement should at least stimulate the patients' inclination to consider this surgical approach and reduce their complaints of postoperative changes in pitch of voice.

The inferior pitch and other parameters in the second postoperative week might be related to an increase of the mass of the vocal folds caused by endotracheal intubation, removal of the enlarged thyroid and bilateral transection of the ST which modified the vascular supply and venous or lymphatic drainage of the larynx. The vocal fold eigenfrequencies primarily determine the $\mathrm{F}_{0}$. Control of eigenfrequencies is largely achieved by varying the stiffness and tension along the anterior-posterior direction of the vocal fold (17). Division of strap muscles might lead to discreet changes in tension and motility of the internal laryngeal muscles, influencing $\mathrm{F}_{0}$ and $\mathrm{F}_{0^{-}}$ high. Additionally, postoperative voice change may also be due to laryngotracheal adhesion of the extralaryngeal musculature (16). Therefore, the delicate movement of the aryngotracheal during the speech was impaired and resulted in the flaw of pitch control. All of these factors may synergistically work on voice recovery.

Lateral intervention, also called the "backdoor approach", was performed between the SCM and strap muscles, indicating secondary or parathyroid surgery. We have described an AA that combines lateral intervention with the "classic" MA and was feasible and effective in handling grossly enlarged glands. It retained the integrity of the ST while still allowing adequate working room and visualization. The key procedure regarding this approach is preserving the ansa cervicalis at the lateral border of the $\mathrm{SH}$ to maintain innervation of the strap muscles, which is further confirmed by nerve stimulation. Anatomically, the ansa cervicalis innervates the ST and SH below the cricoid with a branch from the inferior loop entering the straps within $3 \mathrm{~cm}$ above the sternal notch (7). When the strap musculature was retracted medially from the lateral aspect of the thyroid, the upper lobe was delivered into the immediate surgical field with minimal dissection of superior and retropharyngeal spaces. After safe and individual ligation of the superior pole vessels, EBSLN integrity was checked by stimulating the nerve from the point proximal to the excision of the superior pole vessels. This is definitely applicable in situations where the gland is immersed in inflammatory and fibrotic change, such as Hashimoto's thyroiditis, where there may not be a distinct plane between the medial aspect of the superior thyroid pole and the cricothyroid, which adds to the complexity in their relationship with the EBSLN. In 9 patients examined with large goiters, Cernea showed 13 EBSLNs were at risk and the prevalence of type $2 \mathrm{~b}$ EBSLNs was as high as $54 \%$ (18), which was interpreted as when the gland is of significant size, the superior pole occupies a higher position in the neck and is more closely related to the nerve in its descending course. With lateral access to the upper pole and control of the pedicles located at a much higher level, the EBSLN may come clearly into view. We thus consider this technique of combining lateral intervention with MA to be optimal in thyroid-enlarged cases with the advantage of allowing anterograde identification of EBSLN when having difficulty entering the avascular space, and mobilizing and delivering of the retropharyngeal or deep components into the immediate surgical field without strap muscle division, which aids in post-thyroidectomy voice rehabilitation. The main candidates for this AA are those whose enlarged thyroid extends laterally surpassing the anterior margin of SCM, even reaching the posterior cervical triangle during palpation or CT scanning, and upwardly over the 
level of thyroid cartilage, even reaching the hyoid in CT scanning, and those who are in hope of better conservation of phonatory function. Admittedly, the AA was shown to be time-consuming and much patience was required in preservation of the ST muscles. We showed that it is a patient-friendly technique, but not surgeon-friendly.

To date, little attention is focused on the strap muscle malfunction, the repercussions of which are less serious than those provoked by laryngeal nerve lesions (4). If an open thyroidectomy could be better served by ST cutting, the surgeon would perform it without hesitation. Partial cutting of the ST near its insertion point along the thyroid cartilage is nominated as an efficient technique in exposing the superior pole of the thyroid gland to facilitate exploration and preservation of the EBSLN (19). Furthermore, complete cutting of both the ST and $\mathrm{SH}$ at a lower margin close to the clavicle can reportedly offer an excellent exposure of the surgical field (20). However, though the straps were sutured back to their original position, the denervation or transient ischemia of muscle is inevitable. Despite the controversy on the impact of ST transection on postoperative vocal outcome, we always uphold that "the anatomical structure preserved is the physiological function spared" and believe that it is worthwhile to maximally preserve the tissue property.

Some limitations of this study were noted. As this was a prospective cohort study, the small sample size and lack of control over selection bias weakened the statistical power. According to the sample size analysis, if the true difference of the $\Delta \mathrm{F}_{0}$ between $\mathrm{AA}$ and $\mathrm{MA}$ group is $2.45 \%$, we will need to study at least 51 experimental and 102 control participants, or 68 experimental and 68 control participants, to be able to achieve a power of $90 \%$. Other factors also likely to impact on the postoperative voice such as breath control, scarring and laryngeal movement are difficult to measure objectively, and thus more information should be obtained using electromyography and longer-term follow-up. Some attempts are needed to evaluate the clinical efficacy of anti-adhesive material in the prevention of scar formation among layers of tissues. In summary, although not "simple" from a surgical point of view, this AA for thyroid surgery is realizable, in particular with the use of neuro-stimulator. The AA should be used selectively and developed in the future.

\section{Acknowledgments}

We thank all patients who participated in this study for their cooperation.

Funding: None.

\section{Footnote}

Reporting Checklist: The authors have completed the STROBE reporting checklist. Available at https://dx.doi. org/10.21037/gs-21-510

Data Sharing Statement: Available at https://dx.doi. org/10.21037/gs-21-510

Peer Review File: Available at https://dx.doi.org/10.21037/ gs-21-510

Conflicts of Interest: All authors have completed the ICMJE uniform disclosure form (available at https://dx.doi. org/10.21037/gs-21-510). The authors have no conflicts of interest to declare.

Ethical Statement: The authors are accountable for all aspects of the work in ensuring that questions related to the accuracy or integrity of any part of the work are appropriately investigated and resolved. The study was conducted in accordance with the Declaration of Helsinki (as revised in 2013). The study was approved by Committee on Scientific Research of Shandong University Qilu Hospital (No. 2018149) and informed consent was taken from all individual participants.

Open Access Statement: This is an Open Access article distributed in accordance with the Creative Commons Attribution-NonCommercial-NoDerivs 4.0 International License (CC BY-NC-ND 4.0), which permits the noncommercial replication and distribution of the article with the strict proviso that no changes or edits are made and the original work is properly cited (including links to both the formal publication through the relevant DOI and the license). See: https://creativecommons.org/licenses/by-nc-nd/4.0/.

\section{References}

1. Ravikumar K, Sadacharan D, Muthukumar S, et al. EBSLN and Factors Influencing its Identification and its Safety in Patients Undergoing Total Thyroidectomy: A Study of 456 Cases. World J Surg 2016;40:545-50.

2. Enomoto K, Uchino S, Watanabe S, et al. Recurrent laryngeal nerve palsy during surgery for benign thyroid 
diseases: risk factors and outcome analysis. Surgery 2014;155:522-8.

3. Ching HH, Kahane JB, Foggia MJ, et al. Medial Approach for the Resection of Goiters with Suprahyoid, Retropharyngeal, or Substernal Extension. World J Surg 2018;42:1415-23.

4. Henry LR, Solomon NP, Howard R, et al. The functional impact on voice of sternothyroid muscle division during thyroidectomy. Ann Surg Oncol 2008;15:2027-33.

5. Soylu L, Ozbas S, Uslu HY, et al. The evaluation of the causes of subjective voice disturbances after thyroid surgery. Am J Surg 2007;194:317-22.

6. Butskiy O, Chang BA, Luu K, et al. A systematic approach to the recurrent laryngeal nerve dissection at the cricothyroid junction. J Otolaryngol Head Neck Surg 2018;47:57.

7. Wang RC, Puig CM, Brown DJ. Strap muscle neurovascular supply. Laryngoscope 1998;108:973-6.

8. Aithal VU, Bellur R, John S, et al. Acoustic analysis of voice in normal and high pitch phonation: a comparative study. Folia Phoniatr Logop 2012;64:48-53.

9. Brinca L, Batista AP, Tavares AI, et al. The Effect of Anchors and Training on the Reliability of Voice Quality Ratings for Different Types of Speech Stimuli. J Voice 2015;29:776.e7-14.

10. Chaudhary M, Baisakhiya N, Singh G. Clinicopathological and Radiological Study of Thyroid Swelling. Indian J Otolaryngol Head Neck Surg 2019;71:893-904.

11. Allen E, Minutello K, Murcek BW. Anatomy, Head and Neck, Larynx Recurrent Laryngeal Nerve. Treasure Island, FL. USA: StatPearls publishing, 2021.

12. Sonninen AA. The role of the external laryngeal muscles in

Cite this article as: Zhang B, Lv B, Liu N. Anterolateral approach for thyroid swellings: impact on postoperative voice outcomes. Gland Surg 2021;10(11):3058-3066. doi: 10.21037/ gs-21-510 length-adjustment of the vocal cords in singing; phoniatric, roentgenologic and experimental studies of the mechanism of pitch change in the voice with special reference to the function of the sternothyroid. Acta Otolaryngol Suppl 1956;130:1-102.

13. Niimi S, Horiguchi S, Kobayashi N. F0 raising role of the sternothyroid muscle - an electromyographic study of two tenors. In: Gauffin J, Hammarberg B. editors. Vocal fold physiology: acoustic, perceptual, and physiological aspects of voice mechanisms. Stockholm: Singular Publishing Group Inc., 1991:183-8.

14. Hong KH, Ye M, Kim YM, et al. The role of strap muscles in phonation-in vivo canine laryngeal model. J Voice 1997;11:23-32.

15. Sinagra DL, Montesinos MR, Tacchi VA, et al. Voice changes after thyroidectomy without recurrent laryngeal nerve injury. J Am Coll Surg 2004;199:556-60.

16. Hong KH, Kim YK. Phonatory characteristics of patients undergoing thyroidectomy without laryngeal nerve injury. Otolaryngol Head Neck Surg 1997;117:399-404.

17. Zhang Z. Mechanics of human voice production and control. J Acoust Soc Am 2016;140:2614.

18. Cernea CR, Nishio S, Hojaij FC. Identification of the external branch of the superior laryngeal nerve (EBSLN) in large goiters. Am J Otolaryngol 1995;16:307-11.

19. Lee HS, Kim SW, Park HS, et al. Partial cutting of sternothyroid muscle during total thyroidectomy: impact on postoperative vocal outcomes. ScientificWorldJournal 2013;2013:416535.

20. Ko HY, Lu IC, Chang PY, et al. U-shaped strap muscle flap for difficult thyroid surgery. Gland Surg 2020;9:372-9. 